\title{
Is There a Role for Adjuvant Therapy in RO Resected Gallbladder Cancer?: A Propensity Score-Matched Analysis
}

\section{Se-II Go, MD \\ Young Saing Kim, MD, PhD² \\ In Gyu Hwang, MD, PhD 3 \\ Eun Young Kim, MD, PhD ${ }^{4}$ \\ Sung Yong Oh, MD, $\mathrm{PhD}^{5}$ \\ Jun $\mathrm{Ho} \mathrm{Ji} \mathrm{MD}^{6}$ \\ Haa-Na Song, $\mathrm{MD}^{7}$ \\ Se Hoon Park, MD, $\mathrm{PhD}^{7}$ \\ Joon Oh Park, MD, PhD \\ Jung Hun Kang, MD, $P h D^{1}$}

*A list author's affiliations appears at the end of the paper.

Correspondence: Jung Hun Kang, MD, PhD

Division of Hematology-Oncology,

Department of Internal Medicine,

Institute of Health Sciences,

Gyeongsang National University

School of Medicine, 15 Jinju-daero 816beon-gil,

Jinju 52727, Korea

Tel: 82-55-750-8063

Fax: 82-55-758-9122

E-mail: newatp@naver.com

Co-Correspondence: Joon Oh Park, MD, PhD

Division of Hematology-Oncology,

Department of Medicine,

Samsung Medical Center,

Sungkyunkwan University School of Medicine, 81 Irwon-ro, Gangnam-gu, Seoul 06351, Korea

Tel: 82-2-3410-3459

Fax: 82-2-3410-1754

E-mail: oncopark@skku.edu

Received December 25, 2015

Accepted January 28, 2016

Published Online February 12, 2016

\section{Purpose}

The purpose of this study is to assess the role of adjuvant therapy in stage I-III gallbladder cancer (GBC) patients who have undergone $\mathrm{RO}$ resection.

\section{Materials and Methods}

Clinical data were collected on 441 consecutive patients who underwent RO resection for stage I-III GBC. Eligible patients were classified into adjuvant therapy and surveillance only groups. Propensity score matching (PSM) between the two groups was performed, adjusting clinical factors.

\section{Results}

In total, 84 and 279 patients treated with adjuvant therapy and followed up with surveillance only, respectively, were included in the analysis. Before PSM, the 5-year relapse-free survival (RFS) rate was lower in the adjuvant therapy group than in the surveillance only group (50.8\% vs. $74.8 \%, p<0.001$ ), although there was no statistically significant difference in the 5 -year overall survival (OS) rate $(66.2 \%$ vs. $79.5 \%, p=0.089)$. After the PSM, baseline characteristics became comparable and there were no differences in the 5-year RFS (50.8\% vs. $64.8 \%, p=0.319)$ and $\mathrm{OS}(66.2 \%$ vs. $70.4 \%, p=0.703)$ rates between the two groups.

\section{Conclusion}

The results suggest that fluoropyrimidine-based adjuvant therapy is not indicated in stage I-III GBC patients who have undergone RO resection.

\section{Key words}

Gallbladder neoplasms, Adjuvant chemotherapy, Adjuvant chemoradiotherapy, Survival, Propensity score 


\section{Introduction}

Gallbladder cancer (GBC) is an uncommon disease in Western countries [1]. However, the incidence of GBC is relatively high in Korea, where it accounts for 6.3 per 100,000 individuals and is the sixth leading cause of cancer-related death [2]. The prognosis of GBC is poor, with a 5-year overall survival (OS) rate of $17 \%$ to $45 \%$, even in patients treated with curative intent surgery [3-5]. Advanced stage, highgrade histological differentiation, and a positive resection margin are known to be poor prognostic factors in GBC patients who have undergone surgical resection $[3,6]$.

Given the high relapse rate and poor prognosis, adjuvant therapy would appear to be a rational therapeutic strategy in GBC patients following surgical resection. Nevertheless, there is controversy surrounding the role of adjuvant therapy in GBC. Current National Comprehensive Cancer Network guidelines include consideration of fluoropyrimidine chemoradiotherapy, fluoropyrimidine or gemcitabine chemotherapy, and observation as postoperative options, with no preference given to any particular option [7]. In addition, unlike cholangiocarcinoma, due to the limited clinical data available, the guidelines for GBC do not provide information on treatment strategy according to known prognostic factors, such as resection margin status [7].

Previous studies have suggested that adjuvant therapy may benefit select GBC patients. Several retrospective studies reported that patients with node-positive disease had a survival benefit from adjuvant chemoradiotherapy or radiotherapy $[5,8,9]$. A recent study showed that gemcitabine-based adjuvant chemotherapy improved the survival of GBC patients after R1 or R2 resections [10]. Prospective trials for adjuvant therapy in GBC are extremely rare. Takada et al. [11] reported that GBC patients who have undergone noncurative resections may derive a slight benefit from systemic chemotherapy, but this was not observed in patients who underwent curative surgery in a subgroup analysis of the study, which combined patients with pancreatic, bile duct, and gallbladder cancers.

However, the question of whether patients who have undergone $\mathrm{R} 0$ resection benefit from adjuvant therapy is controversial. A few studies reported that, compared with surgery alone, adjuvant chemoradiotherapy resulted in improved survival in GBC patients following R0 resection $[4,8]$. In contrast, other studies did not show any survival benefit of adjuvant therapy in GBC patients who underwent $\mathrm{R} 0$ resection $[3,11,12]$. Importantly, many previous studies included a small number of patients; heterogeneous populations with respect to tumor location, TNM stage, nodal status, and resection margin; or unbalanced comparative groups. These limitations make it difficult for physicians to identify patients who will truly benefit from adjuvant therapy. A meta-analysis of 20 studies involving 6,712 patients reported that GBC patients who had undergone $\mathrm{R} 1$ resection, but not R0 resection, benefited from adjuvant therapy [13]. Another recent meta-analysis also reported similar results showing that GBC patients who underwent $\mathrm{R} 0$ resection did not benefit from adjuvant therapy compared with surgery alone [14]. However, due to the potential selection bias caused by unbalanced clinical characteristics, which is an inherent drawback of a retrospective study, these two metaanalyses cannot confirm the ineffectiveness of adjuvant therapy in GBC patients who have undergone $\mathrm{R} 0$ resection.

Therefore, we attempted to determine whether adjuvant therapy is necessary for non-metastatic GBC patients who have undergone $\mathrm{R} 0$ resection through a multicenter, casecontrol study with propensity score matching (PSM) to reduce the selection bias.

\section{Materials and Methods}

\section{Study population}

Information on patients who consecutively underwent curative R0 resection for GBC from March 1999 to February 2014 at 6 tertiary medical centers was collected retrospectively. Of the 441 patients who were initially screened, those in the following categories were excluded (Fig. 1): (1) stage IV disease; (2) unknown histological subtype; (3) minor histological subtype such as adenosquamous, mucinous, mucosal, or neuroendocrine carcinoma; (4) follow-up duration less than 3 months; (5) relapse within 1 month of surgical resection; (6) adjuvant therapy with radiotherapy alone; or (7) unknown preoperative serum carbohydrate antigen 19-9 (CA 19-9) level. Ultimately, a total of 363 patients were enrolled in the study. This study was approved by the institutional review board of each participating center.

\section{Treatment}

Curative $\mathrm{R} 0$ resection was performed in all patients and confirmed by the operation record and the pathology report. Then, postoperative management was planned according to each center's protocol. In patients treated with adjuvant chemotherapy alone, fluoropyrimidine-based chemotherapy, including 5-fluorouracil/leucovorin, doxifluridine, tegafur/uracil, or S-1, was performed. Adjuvant concurrent chemoradiotherapy (CCRT) was also performed using fluoropyrimidine-based agents including 5-fluorouracil and capecitabine. Adjuvant chemotherapy and CCRT were initi- 


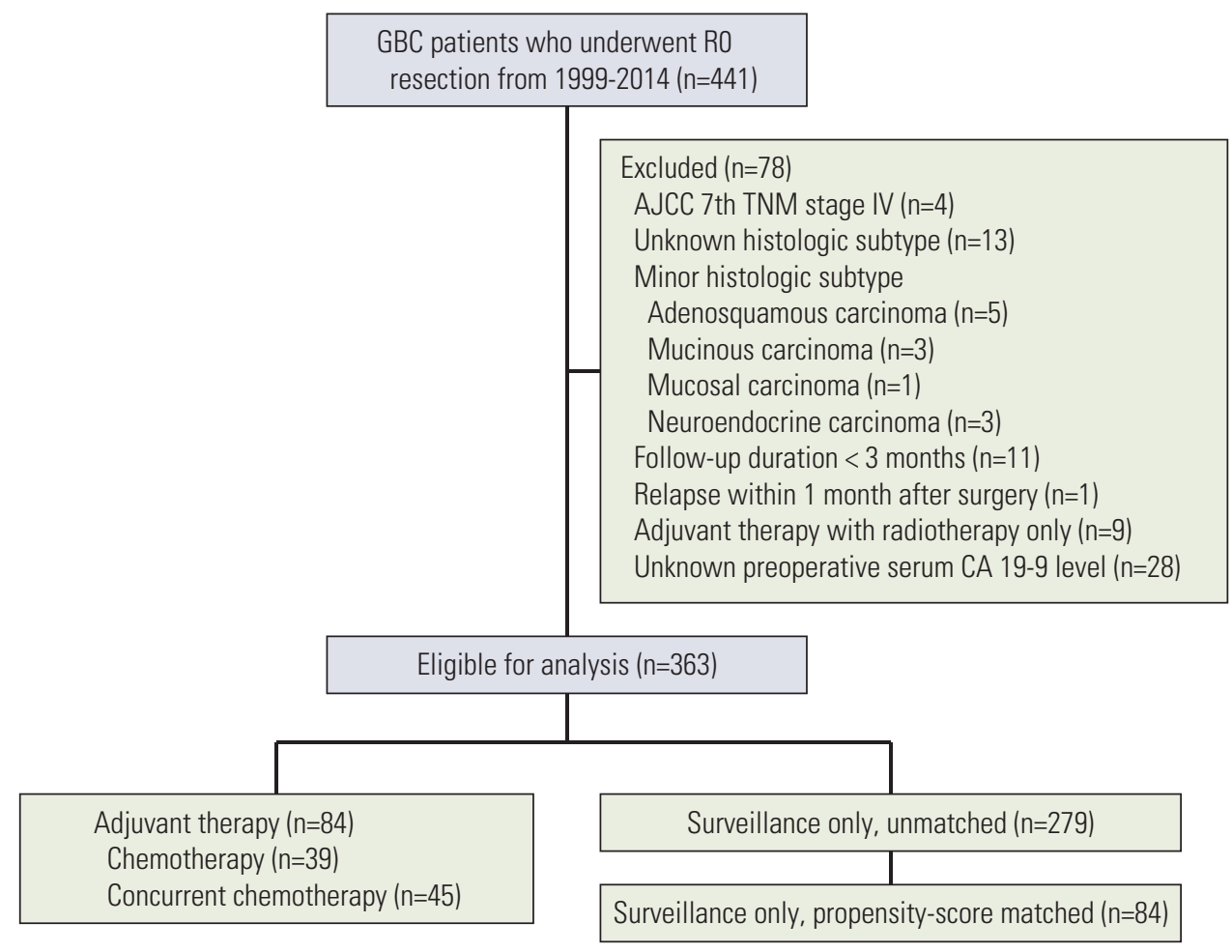

Fig. 1. Study schema. GBC, gallbladder cancer; AJCC, American Joint Committee on Cancer; CA 19-9, carbohydrate antigen $19-9$.

ated within 6 to 8 weeks of surgical resection. Patients managed with surveillance only received follow-up care after surgical resection without adjuvant chemotherapy or CCRT. Regular monitoring was performed in all patients via comprehensive physical examinations, serum tumor markers, and computed tomography for detection of relapse according to each center's protocol.

\section{Data collection}

Clinical data, including demographics, histological type, TNM stage based on the seventh edition of the American Joint Committee on Cancer, preoperative serum level of CA 19-9, treatment modality, and clinical outcomes (e.g., relapse and survival), were collected by medical chart review. The cut-off level of preoperative serum CA 19-9 was defined as $37 \mathrm{U} / \mathrm{mL}$ (the upper limit of the normal range). Relapse-free survival (RFS) was defined as the time from the date of surgery to the date of either the first relapse at any site or death. OS was defined as the time from the date of surgery to the date of death.

\section{Propensity score matching}

We hypothesized that patients with advanced disease may be more likely to be treated with adjuvant therapy at the discretion of the clinician. We therefore performed PSM to reduce the selection bias between patients treated with adjuvant therapy and those managed with surveillance only using the MatchIt package in R statistical software ver. 3.1.3 (The R Foundation for Statistical Computing, Vienna, Austria). The propensity score was calculated from a logistic regression model that included age (years), sex, histological type, TNM stage, and preoperative serum CA 19-9 level (normal or elevated). With the propensity score estimated, 84 pairs of patients in the adjuvant therapy group and the surveillance-only group were matched using a 1:1 nearest neighbor matching algorithm. The standardized mean difference (SMD) in each covariate was calculated to determine whether the PSM was adequately conducted. An SMD less than 0.1 was considered a negligible difference in the mean or prevalence of a covariate between two treatment groups [15].

\section{Statistical analysis}

Statistical comparisons were made using the chi-square 
Table 1. Patient characteristics (total)

\begin{tabular}{|c|c|c|c|c|c|c|c|}
\hline \multirow{2}{*}{ Factor } & \multirow{2}{*}{ Adjuvant therapy ( $n=84)$} & \multicolumn{4}{|c|}{ Surveillance only } & \multirow{2}{*}{ p-value } & \multirow{2}{*}{ SMD } \\
\hline & & Before PSM ( $n=279)$ & p-value & SMD & After PSM (n=84) & & \\
\hline \multicolumn{8}{|l|}{ Age (yr) } \\
\hline$<65$ & $46(54.8)$ & $129(46.2)$ & 0.170 & 0.0324 & $34(40.5)$ & 0.064 & 0.0673 \\
\hline$\geq 65$ & $38(45.2)$ & $150(53.8)$ & & & $50(59.5)$ & & \\
\hline \multicolumn{8}{|l|}{ Sex } \\
\hline Male & $42(50.0)$ & $115(41.2)$ & 0.154 & 0.0439 & $42(50.0)$ & $>0.999$ & 0.0000 \\
\hline Female & $42(50.0)$ & $164(58.8)$ & & & $42(50.0)$ & & \\
\hline \multicolumn{8}{|l|}{ Histological type } \\
\hline W/D and papillary & $31(36.9)$ & $132(47.3)$ & 0.228 & 0.0412 & $32(38.1)$ & 0.781 & 0.0159 \\
\hline $\mathrm{M} / \mathrm{D}$ & $36(42.9)$ & $96(34.4)$ & & & $32(38.1)$ & & \\
\hline $\mathrm{P} / \mathrm{D}$ and $\mathrm{U} / \mathrm{D}$ & $17(20.2)$ & $51(18.3)$ & & & $20(23.8)$ & & \\
\hline \multicolumn{8}{|l|}{ TNM stage } \\
\hline I & $7(8.3)$ & $87(31.2)$ & $<0.001$ & 0.1978 & $7(8.3)$ & 0.949 & 0.0079 \\
\hline II & $34(40.5)$ & $151(54.1)$ & & & $36(42.9)$ & & \\
\hline III & $43(51.2)$ & $41(14.7)$ & & & $41(48.8)$ & & \\
\hline \multicolumn{8}{|l|}{ Nodal status } \\
\hline No & $45(53.6)$ & $245(87.8)$ & $<0.001$ & 0.1712 & $50(59.5)$ & 0.436 & 0.0298 \\
\hline N1 & $39(46.4)$ & $34(12.2)$ & & & $34(40.5)$ & & \\
\hline \multicolumn{8}{|l|}{ CA $19-9(\mathrm{U} / \mathrm{mL})$} \\
\hline$<37$ & $59(70.2)$ & $227(81.4)$ & 0.029 & 0.0556 & $61(72.6)$ & 0.733 & 0.0119 \\
\hline$\geq 37$ & $25(29.8)$ & $52(18.6)$ & & & $23(27.4)$ & & \\
\hline
\end{tabular}

Values are presented as number (\%). PSM, propensity score matching; SMD, standardized mean difference; W/D, well-differentiated; M/D, moderately differentiated; P/D, poorly differentiated; U/D, undifferentiated; CA 19-9, carbohydrate antigen 19-9.

test or Fisher exact test for categorical variables. RFS and OS were estimated using the Kaplan-Meier method, and comparisons between groups were performed using the log-rank test. Multivariate Cox regression analysis using the Enter selection method was performed to identify independent predictors of mortality. All variables with a p-value less than 0.1 in the univariate analysis were entered into the multivariate analysis. The results were reported as hazard ratios (HRs) and $95 \%$ confidence intervals (CIs). A two-tailed p-value less than 0.05 was considered statistically significant. SPSS ver. 21.0 (IBM Co., Armonk, NY) was used for statistical analyses.

\section{Results}

\section{Patient characteristics}

Of the 363 patients included in the study, 84 patients were treated with adjuvant therapy (chemotherapy, $n=39$; CCRT, $\mathrm{n}=45$ ) and 279 were managed with surveillance only after surgical resection. The baseline characteristics before and after PSM are shown in Table 1. In the unmatched cohort, the distributions of age, sex, and histological type were not significantly different between the two groups. However, the proportions of patients with stage III disease (51.2\% vs. $14.7 \%, \mathrm{p}<0.001)$, node-positive disease (46.4\% vs. $12.2 \%$, $\mathrm{p}<0.001)$, and elevated CA $19-9$ levels (29.8\% vs. $18.6 \%$, $\mathrm{p}=0.029$ ) were higher in the adjuvant therapy group than in the surveillance-only group. The SMDs in the TNM stage and nodal status were more than 0.1. After PSM, the SMDs were reduced in all variables except age, and none of the variables showed an SMD of more than 0.1. There were no significant differences in the baseline characteristics between the two treatment groups in the matched cohort (Table 1).

\section{Survival analysis in the unmatched cohort}

In the unmatched cohort, the median follow-up durations were 60.4 and 53.4 months in all patients and in surviving patients at the time of analysis, respectively (range, 5.3 to 177.6 months in both groups). Thirty-seven (44.0\%) and 143 $(51.3 \%)$ patients were followed up for less than 5 years in the adjuvant therapy and surveillance only groups, respectively. 


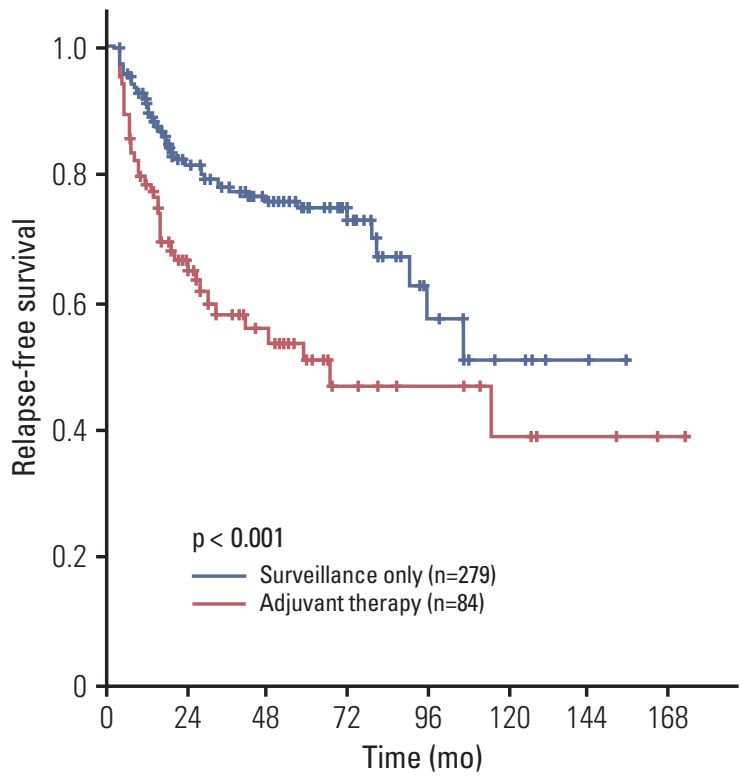

A
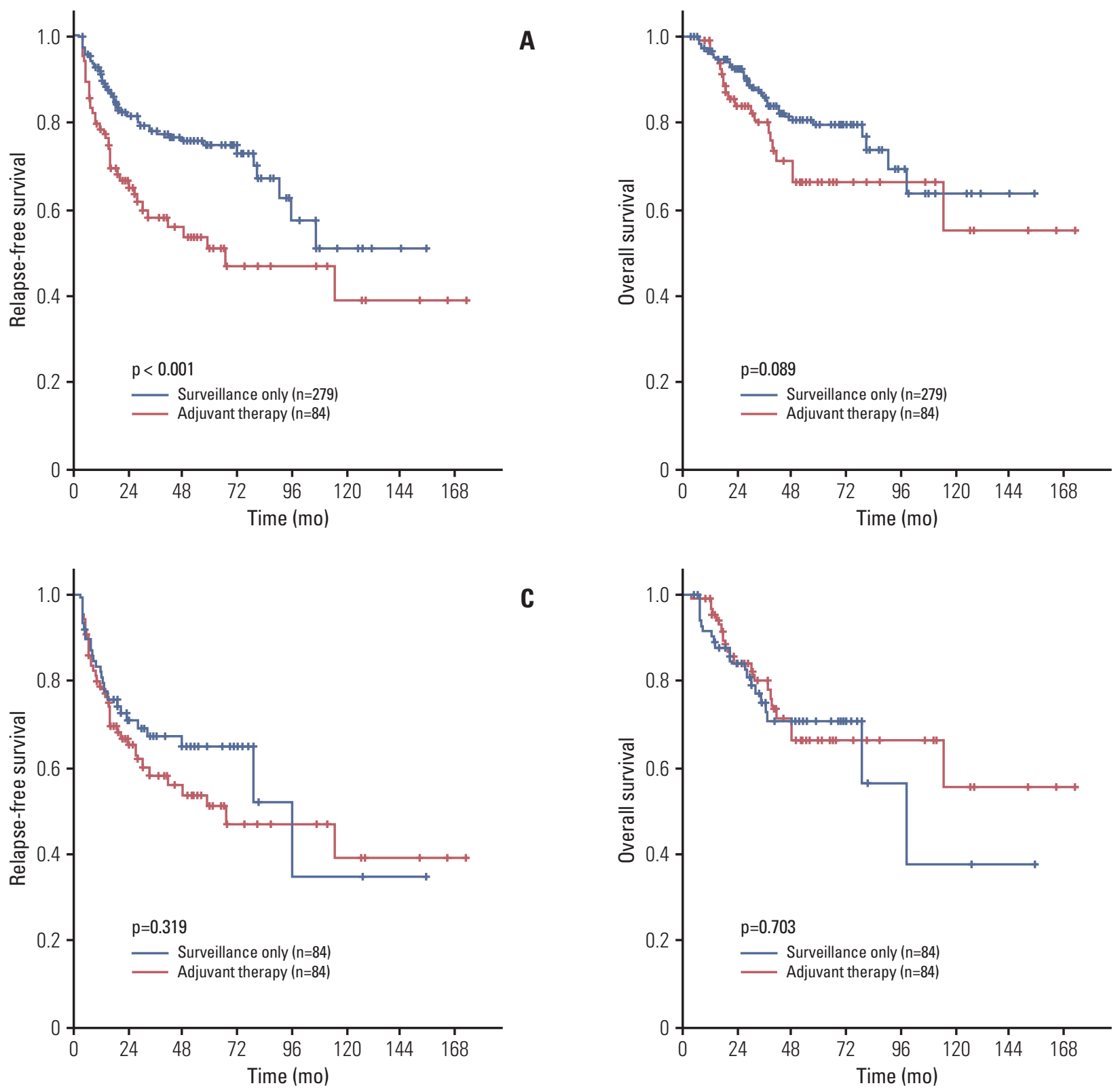

Fig. 2. Kaplan-Meier survival curves according to the type of postoperative treatment. (A) Relapse-free survival before propensity score matching (PSM). (B) Overall survival before PSM. (C) Relapse-free survival after PSM. (D) Overall survival after PSM.

Follow-up was lost in 17 patients $(20.2 \%)$ in the adjuvant therapy group and in 46 patients $(16.5 \%)$ in the surveillance only group. Of the 363 total patients, 85 patients relapsed and 64 patients died. Among the entire cohort, the 5-year RFS and OS rates were $68.9 \%$ and $76.3 \%$, respectively. In comparison of treatment outcomes between the two treatment groups, the adjuvant therapy group showed a shorter RFS compared with the surveillance-only group (3- and 5-year RFS rates, adjuvant therapy $58.0 \%$ and $50.8 \%$ vs. surveillance only $78.0 \%$ and $74.8 \% ; \mathrm{p}<0.001$ ) (Fig. $2 \mathrm{~A}$ ). OS also tended to be worse in the adjuvant therapy group than in the surveillanceonly group (3- and 5-year OS rates, adjuvant therapy 79.9\% and $66.2 \%$ vs. surveillance only $85.5 \%$ and $79.5 \% ; \mathrm{p}=0.089$ ) (Fig. 2B). 
Age (yr)

$\begin{array}{cc}<65 & 80 \\ \geq 65 & 88 \\ \text { Sex } & \\ \text { Male } & 84 \\ \text { Female } & 84\end{array}$

Histologic type

$\begin{array}{ll}\text { W/D and papillary } & 63 \\ \text { M/D } & 68 \\ \text { P/D and U/D } & 37\end{array}$

TNM stage

\begin{tabular}{cc} 
I/II & 84 \\
III & 84 \\
Nodal status & \\
N0 & 95 \\
N1 & 73 \\
\hline
\end{tabular}

CA $19-9(\mathrm{U} / \mathrm{mL})$

\begin{tabular}{rr}
$<37$ & 120 \\
$\geq 37$ & 48 \\
& \\
Total & 168 \\
\hline
\end{tabular}

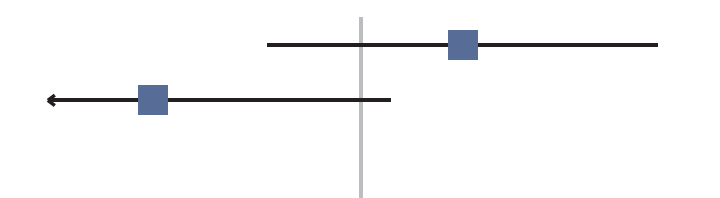

\begin{tabular}{|ll|}
\hline $1.553(0.660-3.652)$ & 0.313 \\
\hline $0.402(0.143-1.132)$ & 0.085 \\
\hline $0.826(0.345-1.977)$ & 0.667 \\
\hline $0.864(0.357-2.090)$ & 0.746 \\
\hline
\end{tabular}

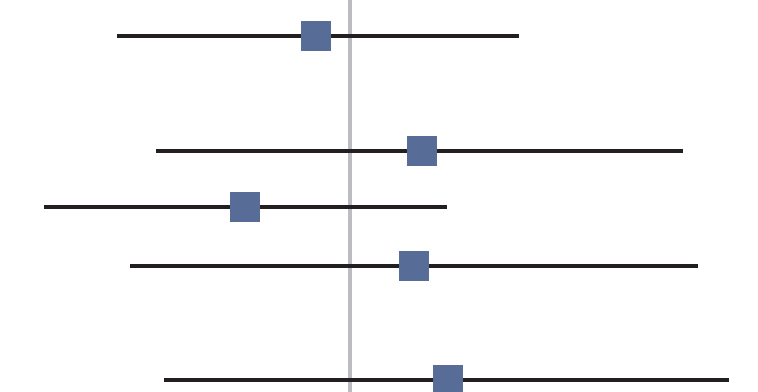

$\begin{array}{ll}1.360(0.429-4.305) & 0.601 \\ 0.629(0.260-1.521) & 0.304 \\ 1.310(0.377-4.559) & 0.671\end{array}$

\begin{tabular}{|ll|}
\hline $1.516(0.438-5.240)$ & 0.511 \\
\hline $0.699(0.344-1.421)$ & 0.322 \\
\hline $1.108(0.397-3.089)$ & 0.844 \\
\hline $0.708(0.332-1.508)$ & 0.370 \\
\hline
\end{tabular}

$\begin{array}{ll}0.903(0.409-1.990) & 0.799 \\ 0.893(0.341-2.339) & 0.817\end{array}$

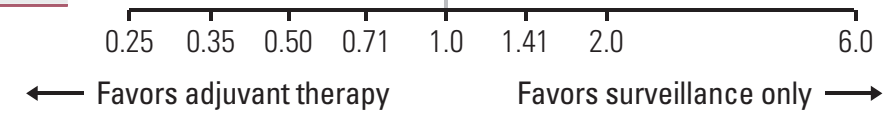

$0.888(0.483-1.633) \quad 0.703$

Fig. 3. Forest plot of subgroup analysis of overall survival. HR, hazard ratio; $\mathrm{CI}$, confidence interval; W/D, well-differentiated; $M / D$, moderately differentiated; $P / D$, poorly differentiated; $U / D$, undifferentiated; $C A$ 19-9, carbohydrate antigen $19-9$.

\section{Survival analysis in the matched cohort}

Further analyses were performed in the matched cohort. The median follow-up durations were 62.1 and 53.4 months in all patients and in surviving patients at the time of analysis, respectively (range, 4.9 to 177.1 months in both groups). Thirty-seven (44.0\%) and $44(52.4 \%)$ patients were followed up for less than 5 years in the adjuvant therapy and surveillance only groups, respectively. Follow-up was lost in 17 patients $(20.2 \%)$ in the adjuvant therapy group and 15 patients $(17.9 \%)$ in the surveillance only group. Among a total of 168 patients, 55 patients relapsed and 42 patients died. In this cohort, RFS was not significantly different between the two treatment groups (3- and 5-year RFS rates, adjuvant therapy $58.0 \%$ and $50.8 \%$ vs. surveillance only $67.1 \%$ and $64.8 \% ; \mathrm{p}=0.319$ ) (Fig. $2 \mathrm{C}$ ). There was also no significant difference in OS between the two treatment groups (3- and 5-year OS rates, adjuvant therapy 79.9\% and 66.2\% vs. surveillance only $74.8 \%$ and $70.4 \% ; \mathrm{p}=0.703$ ) (Fig. 2D). None of the variables, including age, sex, histological type, TNM stage, nodal status, and preoperative serum CA 19-9 level, were associated with the efficacy of adjuvant therapy (Fig. 3). Although there was a tendency in favor of adjuvant therapy in elderly patients, this result was biased by a higher proportion of stage III disease in the surveillance-only group compared with the adjuvant therapy group $(54.0 \%$ vs. $36.8 \%$, $\mathrm{p}=0.110$ ) among elderly patients.

In the matched cohort, advanced TNM stage, higher pre- 
Table 2. Univariate and multivariate analysis of overall survival (total)

\begin{tabular}{|c|c|c|c|c|c|c|}
\hline \multirow{2}{*}{ Factor } & \multicolumn{3}{|c|}{ Univariate } & \multicolumn{3}{|c|}{ Multivariate } \\
\hline & HR & $95 \% \mathrm{CI}$ & p-value & HR & $95 \% \mathrm{CI}$ & p-value \\
\hline Age ( $\geq 65$ yr vs. $<65$ yr) & 0.912 & $0.493-1.686$ & 0.769 & - & - & - \\
\hline Sex (male vs. female) & 0.928 & $0.503-1.711$ & 0.810 & - & - & - \\
\hline Histological type (M/D, P/D, and U/D vs. W/D and papillary) & 1.825 & $0.927-3.594$ & 0.082 & 1.050 & $0.513-2.145$ & 0.895 \\
\hline Stage (III vs. I and II) & 4.361 & $2.167-8.775$ & $<0.001$ & 3.930 & $1.859-8.309$ & $<0.001$ \\
\hline CA $19-9(\geq 37 \mathrm{U} / \mathrm{mL}$ vs. $<37 \mathrm{U} / \mathrm{mL})$ & 2.188 & $1.173-4.084$ & 0.014 & 1.585 & $0.838-2.995$ & 0.156 \\
\hline Tretment (adjuvant therapy vs. surveillance only) & 0.888 & $0.483-1.633$ & 0.703 & 0.837 & $0.454-1.543$ & 0.568 \\
\hline
\end{tabular}

$\mathrm{HR}$, hazard ratio; $\mathrm{CI}$, confidence interval; $\mathrm{M} / \mathrm{D}$, moderately differentiated; $\mathrm{P} / \mathrm{D}$, poorly differentiated; $\mathrm{U} / \mathrm{D}$, undifferentiated; W/D, well-differentiated; CA 19-9, carbohydrate antigen 19-9.

operative serum CA 19-9 level, and histological type other than well-differentiated grade disease were potential poor prognostic factors for OS in the univariate analysis. In the multivariate analysis, advanced TNM stage was the only poor prognostic factor for OS. Adjuvant therapy did not affect the prognosis of this cohort in the multivariate analysis (Table 2).

\section{Survival according to treatment modality in the adjuvant therapy group}

Next, we evaluated the prognosis according to treatment modality among 84 patients treated with adjuvant therapy. Patients treated with chemotherapy had better RFS than those treated with CCRT (3- and 5-year RFS rates, chemotherapy $70.3 \%$ and $57.0 \%$ vs. CCRT $47.3 \%$ and $47.3 \%$; $\mathrm{p}=0.027$ ) (Fig. 4A). However, OS was not significantly different between patients treated with chemotherapy and those treated with CCRT (3- and 5-year OS rates, chemotherapy $87.7 \%$ and $71.0 \%$ vs. CCRT $72.4 \%$ and $62.0 \%$; $=0.203$ ) (Fig. 4B). In addition, patients treated with CCRT were more likely to have poor prognostic characteristics compared to those treated with chemotherapy, as follows: histological type other than well-differentiated grade $(82.2 \%$ vs. $41.0 \%$, $\mathrm{p}<0.001)$, stage III disease ( $80.0 \%$ vs. $17.9 \%, \mathrm{p}<0.001)$, and a higher preoperative serum CA $19-9$ level ( $40.0 \%$ vs. $17.9 \%$, $\mathrm{p}=0.027$ ) (Table 3). In a multivariate analysis adjusted for these poor prognostic markers, CCRT was not associated with worse RFS (HR, 1.501; 95\% CI, 0.685 to 3.286; $\mathrm{p}=0.310$ ) as well as OS (HR, 1.062; 95\% CI, 0.367 to 3.075; $\mathrm{p}=0.912$ ) compared with chemotherapy (Table 4).

\section{Discussion}

Our data suggests that there is no role for adjuvant therapy after $\mathrm{R} 0$ resection in GBC patients. Upon initiating this study, we assessed which patients were likely to be treated with adjuvant therapy after $\mathrm{R} 0$ resection for GBC in clinical practice. Patients with characteristics indicating high tumor burden, such as more advanced TNM stage, node-positive disease, and a higher preoperative serum CA 19-9 level, were more frequently treated with adjuvant therapy compared to those with a low tumor burden. However, adjuvant therapy failed to improve the prognosis of patients with a high tumor burden, resulting in shorter RFS and OS rates compared with the prognosis of patients managed with surveillance only. This paradoxical trend is similar to the results of a large, nonrandomized Japanese study in which, after surgical resection with or without a negative surgical margin, the prognosis of patients who received adjuvant chemotherapy was worse than that of patients who did not receive adjuvant chemotherapy [16]. To minimize the selection bias, PSM was performed in this study. However, even after the characteristics of patients were balanced via PSM, there was no difference in survival, regardless of the postoperative treatment strategy.

Previous studies on adjuvant therapy in GBC patients who have undergone $\mathrm{R} 0$ resection are summarized in Table 5. In accordance with the results of our study, a few studies have suggested that adjuvant therapy is ineffective in GBC patients following R0 resection. A study of 279 GBC patients who had undergone surgery (R0 resection in 164 patients) found that neither adjuvant chemotherapy nor radiotherapy improved the OS of patients following R0 resection [3]. A subgroup analysis of $51 \mathrm{GBC}$ patients who underwent curative resections in a phase III trial showed that they did not benefit from adjuvant chemotherapy [11]. Another recent study of 61 patients with stage III GBC also did not support 

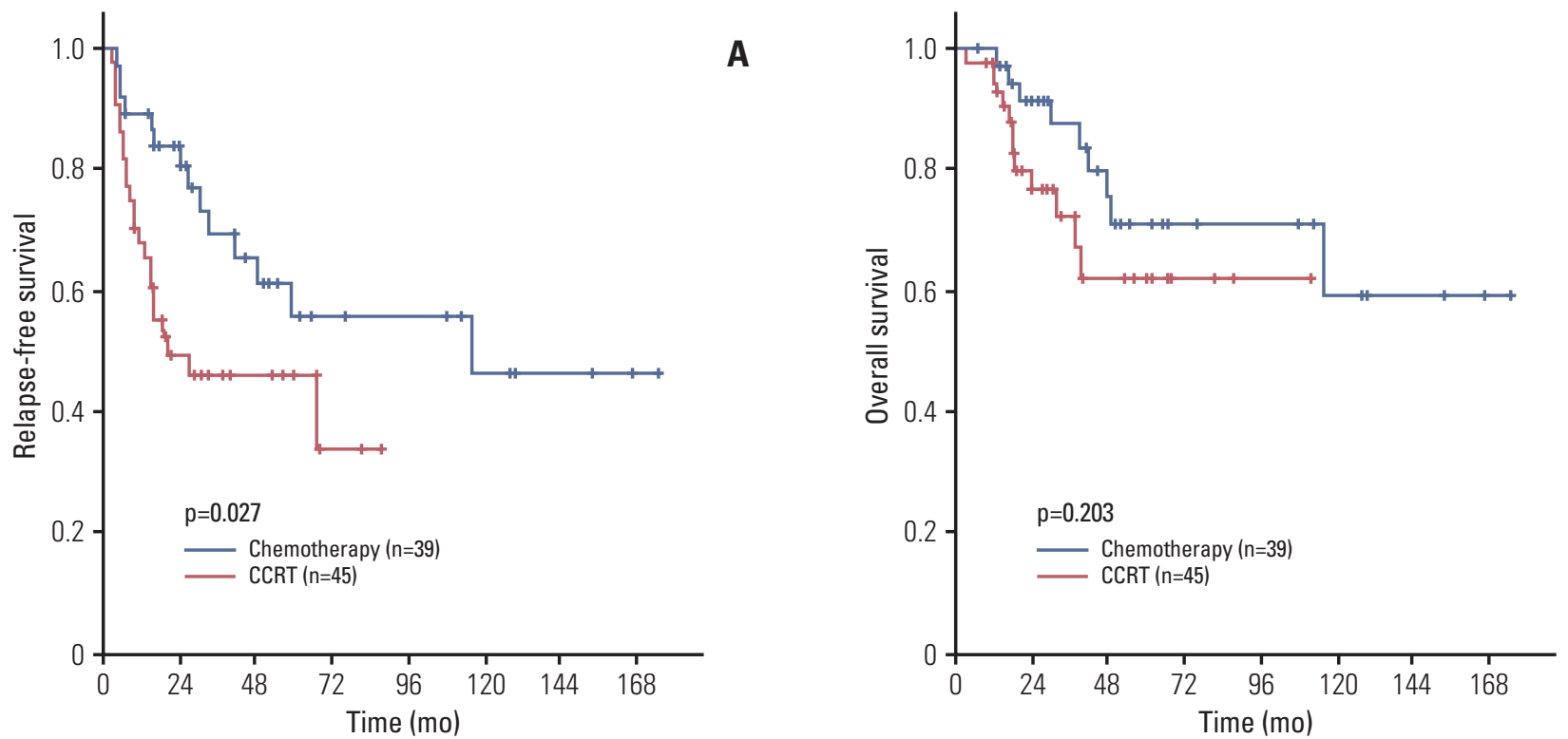

Fig. 4. Kaplan-Meier survival curves according to the type of postoperative treatment (adjuvant therapy group only). (A) Relapse-free survival. (B) Overall survival. CCRT, concurrent chemoradiotherapy.

Table 3. Patient characteristics (adjuvant therapy group only)

\begin{tabular}{|c|c|c|c|}
\hline Factor & Chemotherapy (n=39) & CCRT $(n=45)$ & p-value \\
\hline \multicolumn{4}{|l|}{ Age (yr) } \\
\hline$<65$ & $17(43.6)$ & $29(64.4)$ & 0.055 \\
\hline$\geq 65$ & $22(56.4)$ & $16(35.6)$ & \\
\hline \multicolumn{4}{|l|}{ Sex } \\
\hline Male & $23(59.0)$ & $19(42.2)$ & 0.126 \\
\hline Female & $16(41.0)$ & $26(57.8)$ & \\
\hline \multicolumn{4}{|l|}{ Histological type } \\
\hline W/D and papillary & $23(59.0)$ & $8(17.8)$ & $<0.001$ \\
\hline $\mathrm{M} / \mathrm{D}$ & $11(28.2)$ & $25(55.6)$ & \\
\hline $\mathrm{P} / \mathrm{D}$ and $\mathrm{U} / \mathrm{D}$ & $5(12.8)$ & $12(26.7)$ & \\
\hline \multicolumn{4}{|l|}{ TNM stage } \\
\hline I & $6(15.4)$ & $1(2.2)$ & $<0.001$ \\
\hline II & $26(66.7)$ & $8(17.8)$ & \\
\hline III & $7(17.9)$ & $36(80.0)$ & \\
\hline \multicolumn{4}{|l|}{ Nodal status } \\
\hline No & $34(87.2)$ & $11(24.4)$ & $<0.001$ \\
\hline N1 & $5(12.8)$ & $34(75.6)$ & \\
\hline \multicolumn{4}{|c|}{ Preoperative CA 19-9 (U/mL) } \\
\hline$<37$ & $32(82.1)$ & $27(60.0)$ & 0.027 \\
\hline$\geq 37$ & $7(17.9)$ & $18(40.0)$ & \\
\hline
\end{tabular}

CCRT, concurrent chemoradiotherapy; W/D, well-differentiated; M/D, moderately differentiated; P/D, poorly differentiated; U/D, undifferentiated; CA 19-9, carbohydrate antigen 19-9. 


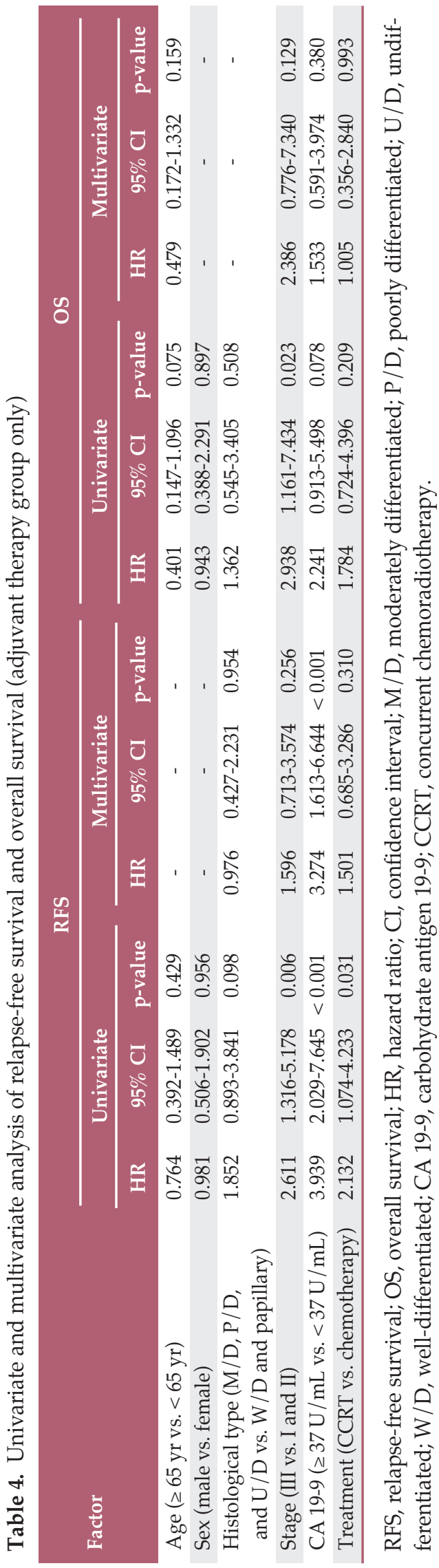

the benefit of adjuvant therapy [12]. However, these previous studies included a small number of patients or enrolled patients who had undergone R1 resection or had stage IV disease, therefore, the results are difficult to interpret. The strengths of our study compared with previous studies are that the role of adjuvant therapy was assessed in selected patients ( $\mathrm{R} 0$ resection, no stage IV disease) and the inevitable selection bias caused by the retrospective design was minimized by PSM. In addition, to the best of our knowledge, our study included the largest number of cases among case-control studies of adjuvant therapy in GBC patients following $\mathrm{R} 0$ resection.

Two previous retrospective studies in GBC patients who had undergone R0 resection yielded opposite results from those obtained in our study. Gold et al. [4] reported that adjuvant chemoradiotherapy reduced the risk of death by $70 \%$ compared with surgery alone in stage I-II GBC patients who had undergone R0 resection. However, the study had a small sample size $(n=73)$, and the prognosis of the surgeryonly group was relatively worse compared with those reported in other studies. For example, in a large Japanese study of 4,770 GBC patients, the 5-year survival rates among the patients in the surgery-only group with stage I and II disease were $78 \%$ and $65 \%$, respectively [16]. Similarly, in our study, the 5-year survival rate was $79.5 \%$ among the patients in the surgery-only group, $85.3 \%$ of whom had stage I or II disease before PSM. In contrast, in the study by Gold et al. [4], the 5-year survival rate in the surgery-only group was approximately $40 \%$, even though $80 \%$ of the patients had stage I disease. This result indicates that the survival difference between patients who underwent surgery alone and those who received adjuvant therapy observed in the study by Gold et al. [4] may be attributed to the poorer prognosis of the surgery-only group compared with those in both the Japanese study as well as our own study. Furthermore, in a previous Western study of over 10,000 cases of GBC based on data from the National Cancer Database (NCDB), the 5 -year survival rates were only 50\% and 29\% among patients with stage I and II disease, respectively, and these trends were identical in the subset of 7,462 patients who underwent potentially curative surgical resection [17]. These findings suggest the possibility of ethnic differences in GBC biology. Cho et al. [8] also reported that adjuvant chemoradiotherapy after surgical resection ( $\mathrm{R} 0$ and $\mathrm{R} 1$ resection in 38 and 2 patients, respectively) is beneficial for node-positive T2/T3 GBC patients. However, the data are difficult to interpret because the sample size was too small and there were few end-point events. Based on both the lack of strong evidence supporting adjuvant therapy and on our findings, we recommend against the routine use of adjuvant therapy in stage I-III GBC patients who have undergone $\mathrm{R} 0$ resection, particularly in Asian patients. 
Table 5. Case-control studies assessing adjuvant therapy in GBC patients following R0 resection

\begin{tabular}{|c|c|c|c|c|c|c|}
\hline Reference & Year & Design & TNM stage & Treatment arma) & No. ${ }^{\text {b) }}$ & Outcome \\
\hline Gold et al. [4] & 2009 & $\mathrm{R}$ & I-II & CRT & 73 & HR for OS: $0.30(p=0.004)$ \\
\hline Cho et al. [8]c) & 2010 & $\mathrm{R}$ & II-III & CRT & 68 & HR for DSS: $0.25(p=0.032)$ in T2/T3N1 disease \\
\hline Takada et al. [11] & 2002 & $\mathrm{P}$ & II-IV & CTx & 51 & 5-Yr survival rate: $46.4 \%$ (CTx) vs. $30.9 \%(\mathrm{~S})(\mathrm{p}=0.1517)$ \\
\hline Park et al. [12] & 2010 & $\mathrm{R}$ & III & CTx, RTx, or CRT & 61 & $\begin{array}{l}\text { 3-Yr survival rate: } 78 \% \text { (CTx), 36\% (RTx), } \\
\text { 36\% (CRT), vs. } 64 \% \text { (S) (p=0.180) }\end{array}$ \\
\hline Lim et al. [3] & 2013 & $\mathrm{R}$ & I-IV & CTx or RTx & 164 & $\begin{array}{l}\text { Adjuvant therapy did not improve survival } \\
\text { (data not shown) }\end{array}$ \\
\hline This study & & $\mathrm{R}$ & I-III & CTx or CRT & 363 & $\begin{array}{l}\text { 5-Yr survival rate in matched sample: } \\
66.2 \%(\mathrm{CTx} \text { or CRT) vs. } 70.4 \%(\mathrm{~S})(\mathrm{p}=0.703) \\
71.0 \%(\mathrm{CTx}) \text { vs. } 62.0 \%(\mathrm{CRT})(\mathrm{p}=0.203)\end{array}$ \\
\hline
\end{tabular}

GBC, gallbladder cancer; R, retrospective; CRT, chemoradiotherapy; HR, hazard ratio; OS, overall survival; DSS, disease-

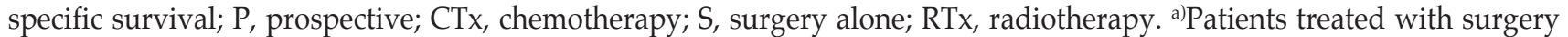
alone are the control arm, ${ }^{b}$ Cases of R1/R2 resection were not included in the analysis except in the study by Cho et al. [8], c) $\mathrm{R} 1$ resection was performed in 6 of 68 patients.

Our study has some limitations in terms of result generalization. First, the study is retrospective in nature. Therefore, the clinical data may not be as accurate as those collected prospectively, and there may be potential selection bias. We tried to control selection bias by using PSM, and patient characteristics were balanced between the groups after PSM. Second, because the data were collected from six institutions, there was heterogeneity in treatment strategies. In this study, however, there was no difference in survival between patients treated with chemotherapy and those treated with CCRT. In addition, all patients enrolled in the study were treated with a fluoropyrimidine-based regimen, mainly due to the national insurance coverage policy in Korea, although the specific dosage and the schedule of chemotherapy and radiotherapy varied. Nevertheless, studies utilizing a uniform treatment strategy are warranted in this setting. Third, the efficacy of adjuvant radiotherapy alone was not assessed in this study due to the small sample size. However, based on the two previous meta-analyses showing that patients receiving adjuvant chemotherapy or chemoradiotherapy derived greater benefit than those receiving radiotherapy alone $[13,14]$, radiotherapy alone may not be recommended as the best option for adjuvant therapy in GBC patients. Fourth, patients with different tumor burden were included together in this study. Despite performance of subgroup analyses by tumor stage and nodal status (Fig. 3), those might be biased because PSM was not done separately for the two variables. Therefore, the association between the tumor burden and the efficacy of adjuvant therapy after R0 resection may not be confirmed in this study. Given the results of previous reports $[13,14]$ and the subgroup analyses of our study (without statistical significance) (Fig. 3) favoring adjuvant therapy in patients with higher tumor stage and node-positive disease, further studies are warranted in these groups with $\mathrm{R} 0$ resection.

\section{Conclusion}

This study suggests that fluoropyrimidine-based adjuvant chemotherapy and CCRT are not indicated in stage I-III GBC patients who have undergone $\mathrm{R} 0$ resection. We suggest that this population be observed or enrolled in clinical trials. Gemcitabine plus cisplatin is the current standard regimen in patients with locally advanced or metastatic biliary tract cancer [18]. Gemcitabine-based adjuvant therapy after curative resection recently showed promising results in patients with biliary tract cancer $[10,19-22]$. Therefore, prospective, randomized studies of therapeutic strategies other than a fluoropyrimidine-based regimen are warranted to improve the prognosis of GBC patients following R0 resection.

\section{Conflicts of Interest}

Conflict of interest relevant to this article was not reported. 


\section{Author Details}

${ }^{1}$ Division of Hematology / Oncology, Department of Internal Medicine, Gyeongsang National University Hospital, Gyeongsang National University School of Medicine, Jinju, ${ }^{2}$ Division of Hematology / Oncology, Department of Internal Medicine, Gachon University Gil Medical Center, Incheon, ${ }^{3}$ Division of Hematology / Oncology, Department of Internal Medicine, Chung-Ang University Hospital, Chung-Ang Uni- versity College of Medicine, Seoul, ${ }^{4}$ Department of Radiology, Gachon University Gil Medical Center, Incheon, ${ }^{5}$ Division of Hematology/Oncology, Department of Internal Medicine, Dong-A University College of Medicine, Busan, ${ }^{6}$ Division of Hematology/Oncology, Department of Internal Medicine, Samsung Changwon Hospital, Sungkyunkwan University School of Medicine, Changwon, 'Division of Hematology-Oncology, Department of Medicine, Samsung Medical Center, Sungkyunkwan University School of Medicine, Seoul, Korea

\section{References}

1. Siegel RL, Miller KD, Jemal A. Cancer statistics, 2015. CA Cancer J Clin. 2015;65:5-29.

2. Jung KW, Won YJ, Kong HJ, Oh CM, Cho H, Lee DH, et al. Cancer statistics in Korea: incidence, mortality, survival, and prevalence in 2012. Cancer Res Treat. 2015;47:127-41.

3. Lim H, Seo DW, Park DH, Lee SS, Lee SK, Kim MH, et al. Prognostic factors in patients with gallbladder cancer after surgical resection: analysis of 279 operated patients. J Clin Gastroenterol. 2013;47:443-8.

4. Gold DG, Miller RC, Haddock MG, Gunderson LL, Quevedo F, Donohue JH, et al. Adjuvant therapy for gallbladder carcinoma: the Mayo Clinic Experience. Int J Radiat Oncol Biol Phys. 2009;75:150-5.

5. Wang SJ, Fuller CD, Kim JS, Sittig DF, Thomas CR Jr, Ravdin PM. Prediction model for estimating the survival benefit of adjuvant radiotherapy for gallbladder cancer. J Clin Oncol. 2008;26:2112-7.

6. Cereda S, Belli C, Reni M. Adjuvant treatment in biliary tract cancer: to treat or not to treat? World J Gastroenterol. 2012; 18:2591-6.

7. National Comprehensive Cancer Network. NCCN Clinical Practice Guidelines in Oncology: hepatobiliary cancers [Internet]. Fort Washington, PA: National Comprehensive Cancer Network; 2015 [cited 2015 Aug 16]. Available from: http:// www.nccn.org/professionals / physician_gls/pdf/hepatobiliary.pdf.

8. Cho SY, Kim SH, Park SJ, Han SS, Kim YK, Lee KW, et al. Adjuvant chemoradiation therapy in gallbladder cancer. J Surg Oncol. 2010;102:87-93.

9. Wang SJ, Lemieux A, Kalpathy-Cramer J, Ord CB, Walker GV, Fuller CD, et al. Nomogram for predicting the benefit of adjuvant chemoradiotherapy for resected gallbladder cancer. J Clin Oncol. 2011;29:4627-32.

10. Nakamura M, Nakashima H, Abe T, Ensako T, Yoshida K, Hino K. Gemcitabine-based adjuvant chemotherapy for patients with advanced gallbladder cancer. Anticancer Res. 2014; 34:3125-9.

11. Takada T, Amano H, Yasuda H, Nimura Y, Matsushiro T, Kato $\mathrm{H}$, et al. Is postoperative adjuvant chemotherapy useful for gallbladder carcinoma? A phase III multicenter prospective randomized controlled trial in patients with resected pancreaticobiliary carcinoma. Cancer. 2002;95:1685-95.

12. Park HS, Lim JY, Yoon DS, Park JS, Lee DK, Lee SJ, et al. Outcome of adjuvant therapy for gallbladder cancer. Oncology. 2010;79:168-73.

13. Horgan AM, Amir E, Walter T, Knox JJ. Adjuvant therapy in the treatment of biliary tract cancer: a systematic review and meta-analysis. J Clin Oncol. 2012;30:1934-40.

14. Ma N, Cheng H, Qin B, Zhong R, Wang B. Adjuvant therapy in the treatment of gallbladder cancer: a meta-analysis. BMC Cancer. 2015;15:615.

15. Austin PC. An introduction to propensity score methods for reducing the effects of confounding in observational studies. Multivariate Behav Res. 2011;46:399-424.

16. Kayahara M, Nagakawa T. Recent trends of gallbladder cancer in Japan: an analysis of 4,770 patients. Cancer. 2007;110: 572-80.

17. Fong Y, Wagman L, Gonen M, Crawford J, Reed W, Swanson $\mathrm{R}$, et al. Evidence-based gallbladder cancer staging: changing cancer staging by analysis of data from the National Cancer Database. Ann Surg. 2006;243:767-71.

18. Valle J, Wasan H, Palmer DH, Cunningham D, Anthoney A, Maraveyas A, et al. Cisplatin plus gemcitabine versus gemcitabine for biliary tract cancer. N Engl J Med. 2010;362: 1273-81.

19. Yamanaka K, Hatano E, Kanai M, Tanaka S, Yamamoto K, Narita $\mathrm{M}$, et al. A single-center analysis of the survival benefits of adjuvant gemcitabine chemotherapy for biliary tract cancer. Int J Clin Oncol. 2014;19:485-9.

20. Ben-Josef E, Guthrie KA, El-Khoueiry AB, Corless CL, Zalupski MM, Lowy AM, et al. SWOG S0809: a Phase II Intergroup Trial of adjuvant capecitabine and gemcitabine followed by radiotherapy and concurrent capecitabine in extrahepatic cholangiocarcinoma and gallbladder carcinoma. J Clin Oncol. 2015;33:2617-22.

21. Murakami Y, Uemura K, Sudo T, Hashimoto Y, Nakashima A, Kondo N, et al. Prognostic factors of patients with advanced gallbladder carcinoma following aggressive surgical resection. 
J Gastrointest Surg. 2011;15:1007-16.

22. Murakami Y, Uemura K, Sudo T, Hashimoto Y, Nakashima A, Kondo N, et al. Prognostic factors after surgical resection for intrahepatic, hilar, and distal cholangiocarcinoma. Ann Surg Oncol. 2011;18:651-8. 